\title{
Tangeretin, a citrus polymethoxyflavonoid, induces apoptosis of human gastric cancer AGS cells through extrinsic and intrinsic signaling pathways
}

\author{
YANG DONG ${ }^{2,3}$, AILI CAO ${ }^{1,2}$, JIANRONG SHI ${ }^{3}$, PEIHAO YIN ${ }^{1}$, LI WANG ${ }^{1}$, \\ GUANG $\mathrm{JI}^{4}$, JIANQUN XIE ${ }^{4}$ and DAZHENG WU ${ }^{1,2}$ \\ ${ }^{1}$ Putuo Hospital, ${ }^{2}$ Institute of Chinese Materia Medica, ${ }^{3}$ Experimental Center, and ${ }^{4}$ Institute of Digestive Diseases, \\ Shanghai University of Traditional Chinese Medicine, Shanghai 200062, P.R. China
}

Received December 2, 2013; Accepted January 27, 2014

DOI: 10.3892/or.2014.3034

\begin{abstract}
Tangeretin, a natural polymethoxyflavone present in citrus peel oil, is known to have anticancer activities in breast cancer, colorectal carcinoma and lung carcinoma, yet, the underlying mechanisms of tangeretin in human gastric cancer AGS cells have not been investigated to date. In the present study, the apoptotic mechanisms of tangeretin in AGS cells were explored. It was observed that tangeretin increased the apoptotic rates of AGS cells following treatment with tangeretin for $48 \mathrm{~h}$ in a dose-dependent manner by Annexin V-FITC and PI double staining. In addition, characteristic apoptotic morphology such as nuclear shrinkage and apoptotic bodies was observed after Hoechst 33258 staining. Flow cytometric assay showed that treatment of AGS cells with tangeretin decreased the mitochondrial membrane potential (MMP) in a dose-dependent manner, which indicated that mitochondrial dysfunction was involved in the tangeretin-induced apoptosis. Caspase-3, -8 and -9 activities were increased by tangeretin in a dose-dependent manner. Western blotting showed that the protein levels of pro-apoptotic proteins including cleaved caspase-3, cleaved caspase-8, cleaved caspase-9, Bax, Bid, tBid, p53, p21/cip1, Fas and FasL were significantly upregulated by tangeretin. In addition, PFT- $\alpha$ (a p53 inhibitor) reduced the apoptotic rates and the expression of p53, p21, caspase- 3 and caspase- 9 induced by tangeretin, indicating that tangeretininduced apoptosis was p53-dependent. In conclusion, these results suggest that tangeretin induces the apoptosis of AGS
\end{abstract}

Correspondence to: Professor Dazheng Wu, Putuo Hospital, Shanghai University of Traditional Chinese Medicine, 164 Lanxi Road, Shanghai 200062, P.R. China

E-mail:wdazheng@vip.sina.com

Professor Jianqun Xie, Institute of Digestive Diseases, Shanghai University of Traditional Chinese Medicine, 725 SouthWanping Road, Shanghai 200032, P.R. China

E-mail: xiejq@gmail.com

Key words: tangeretin, apoptosis, mitochondria, gastric cancer cells mainly through p53-dependent mitochondrial dysfunction and the Fas/FasL-mediated extrinsic pathway.

\section{Introduction}

Tangeretin is a natural polymethoxyflavonoid extracted from citrus fruits (1), which exhibits antiproliferative, anti-invasive, antimetastatic and antioxidant activities (2-4). Recently, many studies have shown that polymethoxylated flavonoids such as tangeretin and nobiletin are more potent inhibitors of tumor cell growth than free hydroxylated flavonoids $(3,5,6)$. Tangeretin inhibits the growth of many cell lines such as breast cancer cell lines MDA-MB-435 and MCF-7, human colon cancer cell line HT-29 and leukaemic HL-60 cells through cell cycle arrest or apoptosis $(2,7,8)$. Tangeretin activates the unfolded protein response and synergizes with imatinib in the erythroleukemia cell line K562 and suppresses IL-1 $\beta$-induced cyclooxygenase (COX)-2 expression through inhibition of p38 MAPK, JNK and AKT activation in human lung carcinoma cells $(9,10)$. In addition, tangeretin was reported to sensitize cisplatinresistant human ovarian cancer cells through downregulation of the phosphoinositide 3-kinase/Akt signaling pathway (11). Thus, tangeretin may be a potential plant-derived agent for use in the treatment of gastric cancer.

Gastric cancer is the second leading cause of cancer-related death in the world (12). AGS is a type of human gastric adenocarcinoma cell line with wild-type p53, which has been used in numerous studies on antineoplastic drugs (13). Wild-type p53 is a tumor-suppressor gene, which plays an important role in cancer cell growth inhibition and mediates either cell cycle arrest or apoptosis $(14,15)$. In response to various forms of stress, p53 is activated and accumulates in the nucleus, where it regulates the transcription of numerous target genes using specific DNA response elements. Beyond its nuclear role, p53 regulates a number of mitochondrial functions. However, the mutant p53 existing in some tumor cells loses its activity and has no antitumor function (16). Thus, as a gastric cancer cell line with wild-type p53, AGS is suitable for investigation of the role of $\mathrm{p} 53$ in the anticancer action of tangeretin.

Our previous study showed that tangeretin inhibits the proliferation of human AGS gastric cancer cells and induces 
cell cycle arrest in the $\mathrm{S}$ and $\mathrm{G} 2 / \mathrm{M}$ phases through inhibition of ERK phosphorylation and upregulation of cyclin B1 protein (17). In the present study, we further examined the effects of tangeretin on the apoptosis of AGS cells and explored the related mechanisms.

\section{Materials and methods}

Reagents and antibodies. Tangeretin was obtained from Shanghai Tongtian Biotechnology Co., Ltd. (Shanghai, China). Methylthiazolyldiphenyl-tetrazolium bromide (MTT) was obtained from Shanghai Richu Biotechnology Ltd. Propidium iodide (PI) was from Sigma-Aldrich, Inc. (St. Louis, MO, USA). ECL Plus Western Blotting detection reagents were from GE Healthcare (Wauwatosa, WI, USA). Annexin V-FITC apoptosis kit and caspase-3, $-8,-9$ colorimetric assay kits were obtained from BioVision (Mountain View, CA, USA). All antibodies and secondary antibodies were from Cell Signaling Technology (Beverly, MA, USA).

Cell culture. Human gastric cancer cell line AGS from the American Type Culture Collection (ATCC, Rockville, MD, USA) was cultured in HAM's/F12 medium (HyClone, Logan, UT, USA) supplemented with $10 \%$ fetal bovine serum (FBS) at $37^{\circ} \mathrm{C}$ in a $5 \% \mathrm{CO}_{2}$ air atmosphere.

MTT assay for cell viability. Cells were seeded in 96-well plates and treated with different concentrations of tangeretin for 24, 48 and $72 \mathrm{~h}$, respectively. Then, MTT solution was added to each well at a final concentration of $1 \mathrm{mg} / \mathrm{ml}$, and the plates were incubated at $37^{\circ} \mathrm{C}$ for another $4 \mathrm{~h}$. After incubation, $150 \mu 1$ dimethyl sulfoxide (DMSO) was added to each well and the absorbance was read at $570 \mathrm{~nm}$ using an absorbance microplate reader (SpectraMax 190; Molecular Devices, Sunnyvale, CA, USA). The experiments were performed in triplicate and at least repeated three times. Inhibition rates $=(1-$ sample OD/ control OD) x $100 \%$.

Analysis of apoptosis. Apoptosis was evaluated using an Annexin V-FITC and PI double staining kit. After treatment with 10,30 and $60 \mu \mathrm{M}$ tangeretin or $0.05 \%$ DMSO for $48 \mathrm{~h}$, AGS cells were harvested and incubated with $500 \mu \mathrm{l}$ Annexin V binding buffer containing $5 \mu \mathrm{g} / \mathrm{ml}$ PI and $1 \mu \mathrm{l}$ FITC-labeled Annexin V for $30 \mathrm{~min}$ on ice. Then cells were analyzed by a FACSCalibur flow cytometer (BD Biosciences, San Jose, CA, USA), and the results were indicated as percentages of apoptotic cells. Images of cells were captured under an inverted fluorescence microscope (IX71, Olympus, Tokyo, Japan).

Hoechst 33258 staining. After treatment with tangeretin for $48 \mathrm{~h}$, cells were fixed in $4 \%$ paraformaldehyde for $2 \mathrm{~h}$ and stained with $20 \mu \mathrm{M}$ Hoechst 33258 for 20 min after washing with PBS. Cells were then observed and images were captured under an inverted fluorescence microscope.

Caspase-3, -8 and -9 activity assay. Caspase activity was examined by a caspase- $3,-8,-9$ colorimetric assay kit respectively according to the manufacturer's protocol. Sample readings at $405 \mathrm{~nm}$ were conducted using a SpectraMax 190

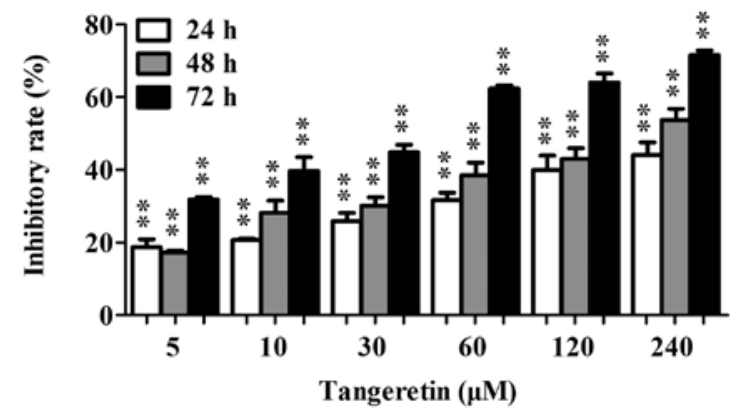

Figure 1. Effect of tangeretin on AGS cell viability. MTT assays showed that tangeretin inhibited the growth of AGS cells in a dose- and time-dependent manner. Data are presented as mean \pm SEM, $n=6 ;{ }^{* *} \mathrm{P}<0.01$ vs. control.

microplate reader. Fold-increase in activity was determined by comparing the results with the level of the control.

Mitochondrial transmembrane potential assay. The cells treated with tangeretin were collected by centrifugation at $3,000 \mathrm{rpm}$ for $10 \mathrm{~min}$ and suspended in $500 \mu \mathrm{l}$ PBS. The cells were incubated with Rhodamine $123(1 \mu \mathrm{g} / \mathrm{ml})$ for $30 \mathrm{~min}$ and examined by a FACSCalibur flow cytometer.

Western blot analysis. After a 48-h exposure to tangeretin at different doses, cells were washed twice with ice-cold PBS and lysed in protein lysis buffer. Protein concentrations were tested by the Bradford method. Proteins were subjected to electrophoresis on $12 \%$ polyacrylamide gels and then transferred to polyvinylidene difluoride (PVDF) membranes by electrotransfer (Bio-Rad Laboratories, CA, USA). After blocking with 5\% non-fat milk in PBS buffer containing 0.5\% Tween-20 and washed three times, the membranes were incubated with various primary antibodies, respectively. Membranes were washed and incubated with the corresponding secondary antibodies, and then the protein bands were visualized with ECL Plus Western blotting detection reagents.

Statistical analysis. Data are presented as means \pm SEM. Statistical analysis of data was carried out with GraphPad Prism 5.0 (Hearne Scientific Software, Chicago, IL, USA). Multiple comparisons were carried out by one-way analysis of variance (ANOVA). A value of $\mathrm{P}<0.05$ was considered to indicate a statistically significant result.

\section{Results}

Effects of tangeretin on the viability of AGS cells. The antiproliferative effect of tangeretin on the human gastric cancer AGS cell line was examined by MTT assay. The inhibitory rates of the cells exposed to 5,10,30,60, 120 and $240 \mu \mathrm{M}$ tangeretin for 24,48 and $72 \mathrm{~h}$ were observed, respectively. The results showed that tangeretin decreased the viability rates of AGS cells in a dose- and time-dependent manner (Fig. 1).

Tangeretin induces AGS cell apoptosis. Flow cytometric assay showed that the early apoptosis rates and late apoptosis (necrosis) rates of AGS cells treated with tangeretin for $48 \mathrm{~h}$ increased in a dose-dependent manner (Fig. 2A; 

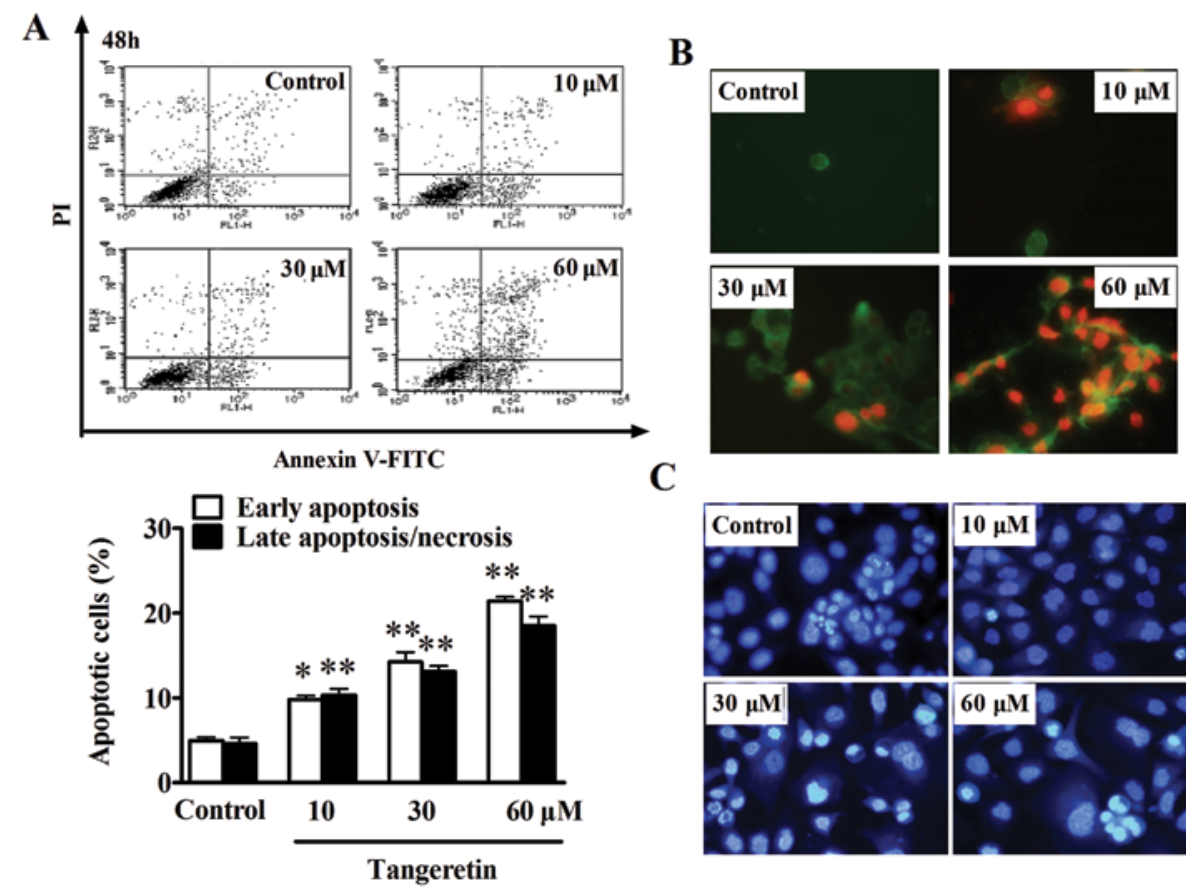

C

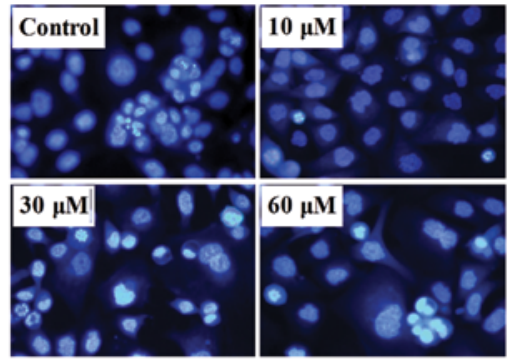

Figure 2. Effect of tangeretin on AGS cell apoptosis. (A) Flow cytometric assay showed that the percentages of early and late apoptotic cells increased in a dose-dependent manner as determined by Annexin V-FITC/PI staining. Data are presented as means \pm SEM, $n=3$; ${ }^{*} \mathrm{P}<0.05$, ${ }^{* *} \mathrm{P}<0.01$ vs. control. (B) The percentages of Annexin V-FITC (green)-labeled early apoptotic cells and PI (red)-labeled late apoptotic or necrotic cells increased with the rise in the concentration of tangeretin. (C) Characteristic apoptotic morphology including cell nuclear shrinkage, chromatin condensation and apoptotic bodies was observed following Hoechst 33258 staining.
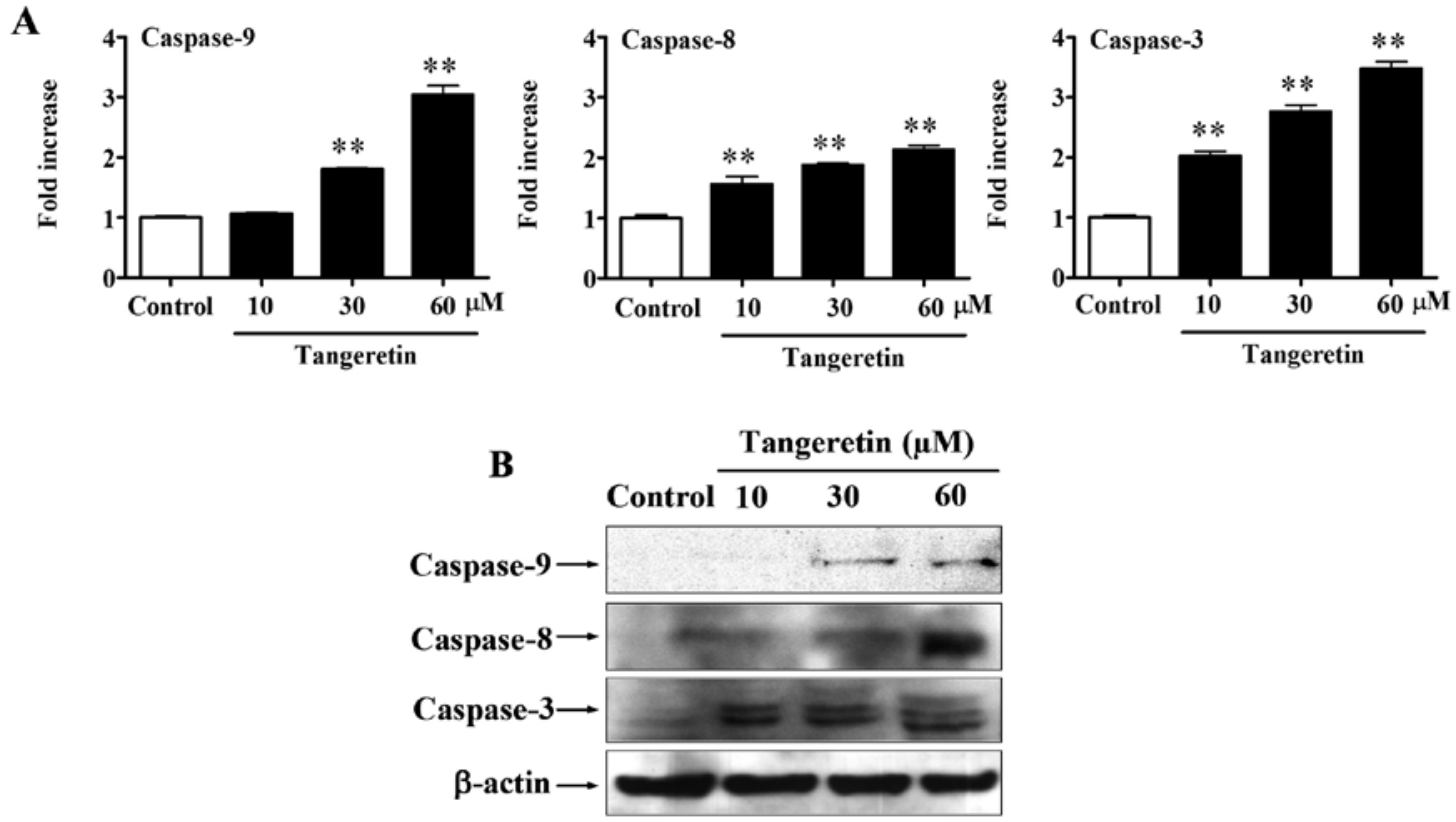

Figure 3. Effect of tangeretin on caspases. (A) Tangeretin increased the activity of caspase-3, -8 and -9 in AGS cells in a dose-dependent manner. Data are presented as means \pm SEM, $n=3 ;{ }^{* *} \mathrm{P}<0.01$ vs. control. (B) Western blot analysis showed that levels of cleaved caspase $-3,-8$ and -9 were increased following treatment with tangeretin in a dose-dependent manner.

$\mathrm{P}<0.05, \mathrm{P}<0.01)$. Fig. $2 \mathrm{~B}$ also shows that the percentage of Annexin V-FITC (green)-labeled early apoptotic cells and PI (red)-labeled late apoptotic or necrotic cells increased along with the increase in the concentration of tangeretin. In addition, typical apoptotic morphological changes including cell nuclear shrinkage, chromatin condensation and apoptotic bodies were observed after exposure to different doses of tangeretin by Hoechst 33258 staining (Fig. 2C). These results confirmed that tangeretin induced the apoptosis of AGS cells.

Involvement of caspases in tangeretin-induced apoptosis. As shown in Fig. 3A, the results showed that the activities of 

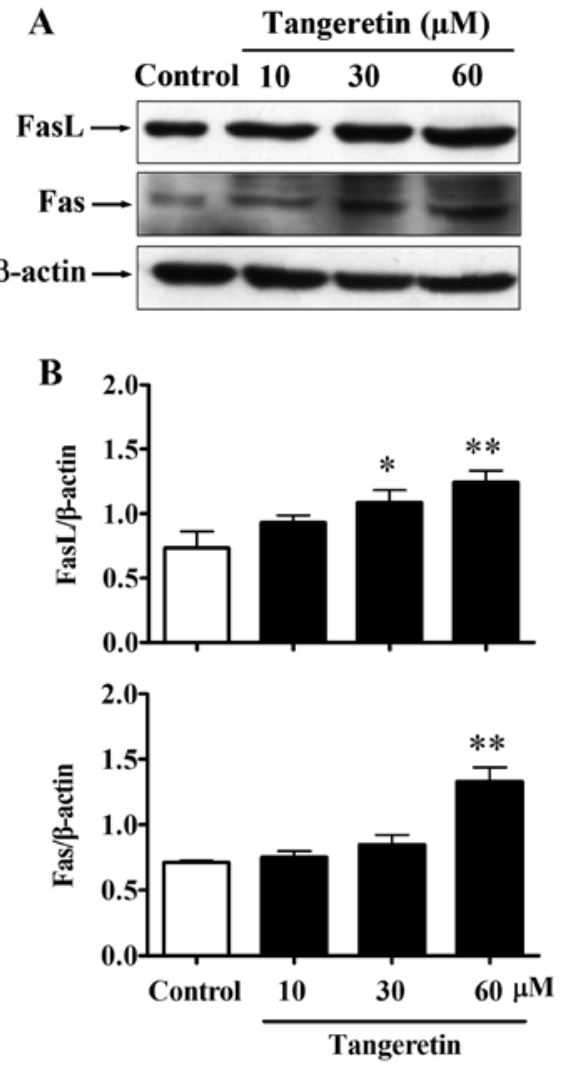

Figure 4. Effect of tangeretin on the levels of Fas and FasL expression. Western blot analysis showed that the expression levels of Fas and FasL increased in AGS cells following treatment with tangeretin for $48 \mathrm{~h}$. Data are presented as means \pm SEM, $n=4-6 ;{ }^{*} \mathrm{P}<0.05,{ }^{* *} \mathrm{P}<0.01$ vs. control.

caspase-3, -8 and -9 were increased by tangeretin in a dosedependent manner after a 48 -h exposure $(\mathrm{P}<0.01)$. Treatment of cells with $60 \mu \mathrm{M}$ tangeretin increased the caspse-3 activity $\sim 3.5$-fold, caspase- $8 \sim 1.8$-fold and caspase-9 $\sim 3.0$-fold Western blot assay showed that the expression levels of cleaved caspase- $3,-8$ and -9 proteins were upregulated by tangeretin in a dose-dependent manner (Fig. 3B). These results indicate that tangeretin induces both the extrinsic and intrinsic apoptotic pathways with the activation of the caspase cascade.

Fas/FasL is involved in tangeretin-induced apoptosis. To examine the extrinsic signaling pathway through Fas, the expression levels of death receptor Fas and its ligand FasL were assessed. As shown in Fig. 4, the expression of Fas and FasL was increased in a dose-dependent manner $(\mathrm{P}<0.05, \mathrm{P}<0.01)$. Thus, the binding of FasL to Fas induced Fas trimerization, which recruited and activated initiator caspase-8. Activated caspase- 8 stimulates apoptosis via two parallel cascades; it directly cleaves and activates caspase-3, or it cleaves the proapoptotic Bcl-2 family protein Bid. Cleaved/truncated Bid (tBid) translocates to the mitochondria, inducing cytochrome $c$ release, which sequentially activates caspase- 9 and caspase- 3 .

Mitochondrial signaling pathway in tangeretin-induced apoptosis. Decrease and breakage of mitochondrial membrane potential (MMP) is an important sign of mitochondrial damage. Flow cytometric assay after Rhodamine 123 staining showed that the percentages of cells with high MMP decreased in a
A
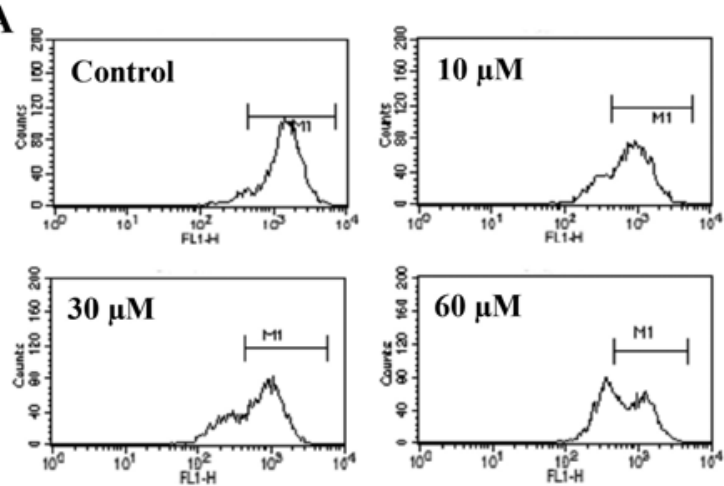

B

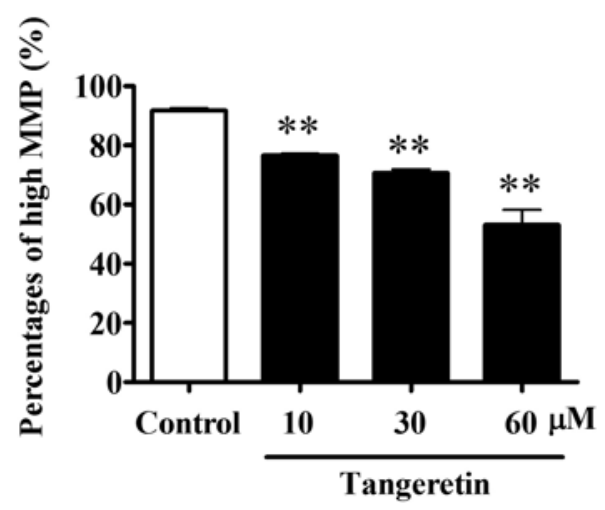

Figure 5. Effect of tangeretin on mitochondrial membrane potential (MMP) of AGS cells. (A) Following treatment with 10,30 and $60 \mu \mathrm{M}$ tangeretin for $48 \mathrm{~h}$, cells stained with Rhodamine 123 were examined by flow cytometry. (B) Tangeretin decreased the percentages of AGS cells with high MMP. Data are presented as means $\pm \mathrm{SEM}, \mathrm{n}=4 ;{ }^{* *} \mathrm{P}<0.01$ vs. control.

dose-dependent manner $(\mathrm{P}<0.01)$, suggesting that tangeretin reduced the levels of MMP and induced mitochondrial damage (Fig. 5). In addition, the protein levels of cytochrome $c$, Bax, Bid and tBid increased as the dose of tangeretin increased (Fig. 6; $\mathrm{P}<0.05, \mathrm{P}<0.01$ ). These results indicate that Bax and tBid activated by upstream proteins mediated mitochondrial dysfunction, induced the release of cytochrome $c$ and activation of caspase- 9 and caspase-3. In addition, the extrinsic signaling pathway interacted with the mitochondrial signaling pathway through induction of cleavage of Bid by caspase- 8 .

Tangeretin-induced apoptosis is dependent on p53 activation. As shown in Fig. 7A, significant elevations in the p53 and $\mathrm{p} 21$ protein levels were noted in a dose-dependent manner $(\mathrm{P}<0.01)$. To determine whether tangeretin-induced apoptosis is p53-dependent, a p53 inhibitor Pifithrin- $\alpha$ (PFT- $\alpha$ ) was used. The results showed that $20 \mu \mathrm{M}$ PFT- $\alpha$ reduced the apoptotic rates induced by tangeretin from $15.8 \pm 0.1$ to $7.6 \pm 1.1 \%$ (Fig. 7B; $\mathrm{P}<0.01, \mathrm{P}<0.001$ ). In addition, western blot analysis showed that $20 \mu \mathrm{M}$ PFT- $\alpha$ reduced the expression of $\mathrm{p} 53$, p21, caspase-3 and caspase-9 proteins significantly increased by $60 \mu \mathrm{M}$ tangeretin (Fig. 7C). These results suggest that tangeretin-induced apoptosis of AGS cells was p53-dependent.

\section{Discussion}

The present study showed that tangeretin inhibited the viability and induced the apoptosis of human gastric cancer 

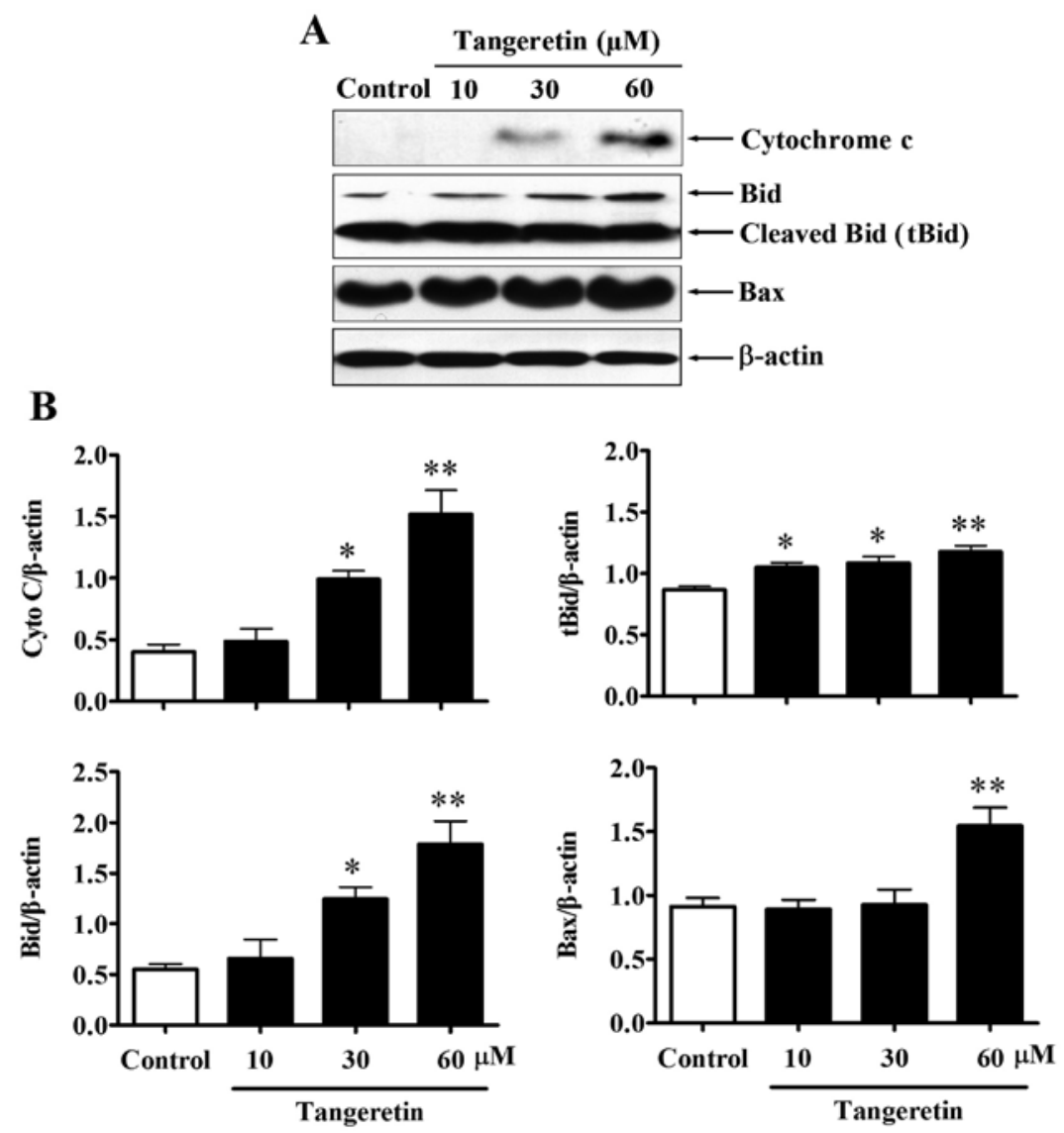

Figure 6. Effect of tangeretin on the expression levels of pro-apoptotic proteins in AGS cells. (A and B) Western blot assay showed that protein expression levels of cytochrome $c$, Bid, tBid and Bax were increased following tangeretin treatment for $48 \mathrm{~h}$ in a dose-dependent manner. Each column in B represents the means \pm SEM, $n=3 ;{ }^{*} \mathrm{P}<0.05,{ }^{* *} \mathrm{P}<0.01$ vs. control.

A
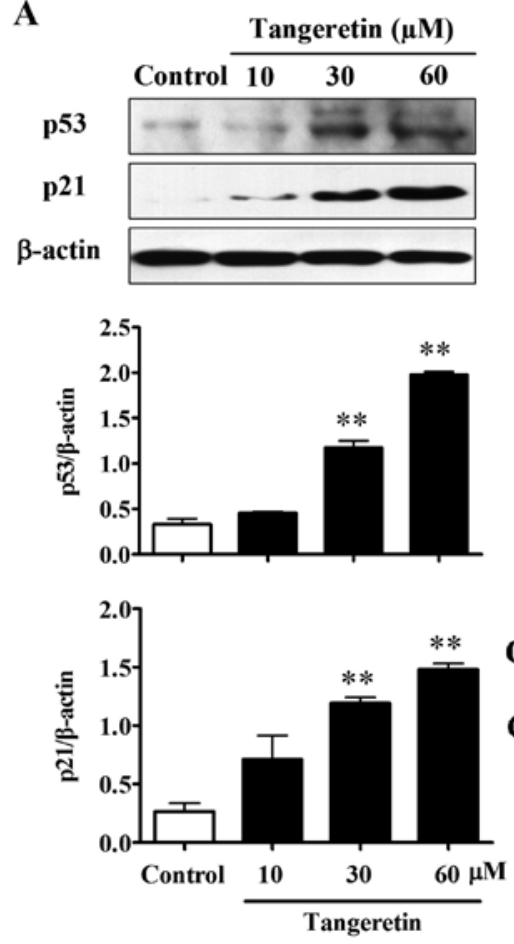

B

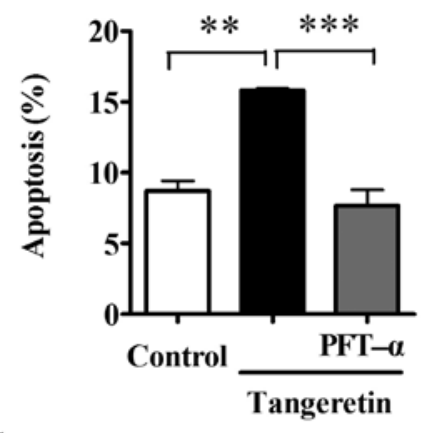

C

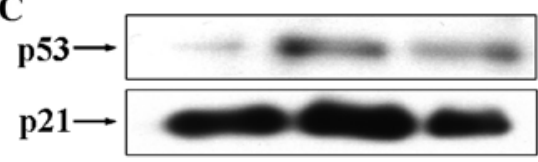

Caspase-9 $\longrightarrow \longrightarrow$

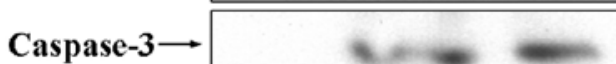

$\beta$-actin $\rightarrow$

Tangeretin

Figure 7. The p53-dependent pathway in tangeretin-induced apoptosis in AGS cells. (A) Western blot analysis showed that p53 and p21 expression in AGS cells was increased in a dose-dependent manner following treatment with tangeretin. (B) PFT- $\alpha$ (a p53 inhibitor) reduced the apoptotic rates of AGS cells induced by $60 \mu \mathrm{M}$ tangeretin. (C) Western blot analysis showed that PFT- $\alpha$ inhibited tangeretin-induced elevation of p53, p21, caspase-3 and caspase-9 expression levels. Each column represents the means $\pm \mathrm{SEM}, \mathrm{n}=3 ;{ }^{*} \mathrm{P}<0.05,{ }^{* * *} \mathrm{P}<0.01,{ }^{* * * *} \mathrm{P}<0.001$ vs. control. 


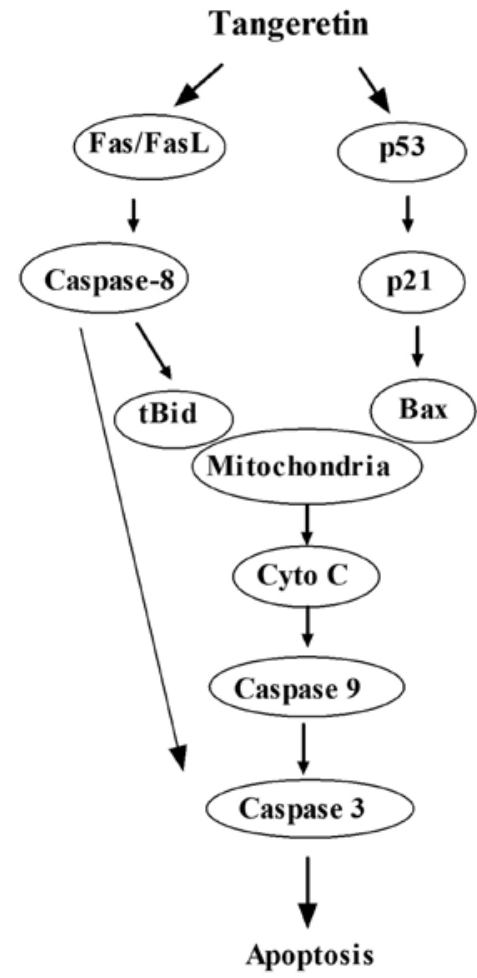

Figure 8. Pathway of tangeretin-induced apoptosis of AGS cells.

AGS cells through both extrinsic and intrinsic signaling pathways through the activation of the caspase cascade. The present study provides important data to support the finding that tangeretin as a natural component widely existing in many fruits or fruit juices exhibits antineoplastic activity in human gastric cancer.

Caspases play a crucial role in apoptosis, and the activation of caspases amplifies apoptotic signaling (18). In the present study, tangeretin increased the activities of caspase- $3,-8$ and -9 and upregulated the expression of cleaved caspase- $-3,-8$ and -9 proteins. The active forms of caspase -8 and -9 can activate downstream effectors caspase-3, -6 and -7 , resulting in the cleavage of several intracellular polypeptides (e.g., PARP-1) as well as activation of DNase, which leads to DNA fragmentation (19).

Apoptosis is mainly mediated by the death receptor-triggered extrinsic pathway, the mitochondrial-initiated intrinsic pathway and the endoplasmic reticulum pathway (20-22). The dissipation of MMP marks the point of no return during the apoptotic program and occurs prior to DNA fragmentation. Thus, the evaluation of MMP depolarization is of critical importance for the assessment of apoptosis $(23,24)$. In the present study, MMP was observed to be reduced by tangeretin in a dose-dependent manner, which suggests that mitochondrial dysfunction contributed to the tangeretin-induced apoptosis. In addition, western blot analysis showed that the expression levels of Fas, FasL, Bid and tBid were increased by tangeretin. It was previously reported that Bid is activated through cleavage by caspase- 8 to become cleaved Bid (tBid) in the Fas-induced process of apoptosis, and tBid is necessary for Bax oligomerization (25-27). Therefore, in the present study, tangeretin may have activated caspase- 8 through Fas/
FasL, and then the activated caspase- 8 cleaved Bid into tBid, which was then transferred to the mitochondria where it probably combined with Bax. Then cytochrome $c$ was released to activate caspase-9 and caspase-3 (Fig. 8).

p53 is a transcription factor which plays an important role in the cellular response to DNA damage through either cell apoptosis or cell cycle arrest $(13,28,29)$. In our previous study, tangeretin induced $\mathrm{S}$ phase arrest at $24 \mathrm{~h}$ and G2/M arrest at $48 \mathrm{~h}$ in AGS cells (17,30). In the present study, the results showed that p53 expression was elevated by tangeretin in a dose-dependent manner associated with upregulation of p2 $1^{\text {waf } 1 / \text { cip } 1}$ and Bax in AGS cells. p21 plays an essential role in growth arrest after DNA damage, and its overexpression leads to $\mathrm{G} 1$ and $\mathrm{G} 2$ phase cell cycle arrest (31). Upregulation of p21 $1^{\text {waf1/cipl }}$ is also involved in the modulation of apoptosis in gastric cancer cells (13). Bax expression can be regulated by p53 (32). To investigate the function of p53 in tangeretin-treated AGS cells, we observed the effect of a p53 inhibitor (PFT- $\alpha$ ) on the rate of apoptosis of tangeretin-treated AGS cells. The results showed that PFT- $\alpha$ reduced the apoptotic rate of AGS cells following treatment with tangeretin, which confirmed that the apoptosis induced by tangeretin was p53-dependent. Western blot analysis showed that PFT- $\alpha$ inhibited the expression of $\mathrm{p} 21$, Bax, caspase-3 and caspase-9, which indicated that p53 was located upstream of these proteins in the apoptotic pathway. Thus, these results suggest that tangeretin induces the apoptosis of AGS cells by p53-activated Bax. Then Bax acts in the mitochondria to cause the release of cytochrome $c$, leading to the activation of caspase- 9 , and the subsequent activation of caspase-3.

In summary, as shown in Fig. 8, tangeretin induced the apoptosis of AGS cells through extrinsic and intrinsic signaling pathways. Activation of p53 induced mitochondrial-mediated apoptosis through the upregulation of Bax, which contributed to the activation of caspase-9, leading to the activation of downstream caspases in the process. Moreover, the Fas/ FasL-mediated death receptor pathway may interact with the mitochondrial signaling pathway through caspase-8-cleaved Bid.

\section{Acknowledgements}

The present study was supported by the National Science and Technology Major Project of China (2009ZX09311-003); the Project of Shanghai Leading Academic Discipline, Shanghai Education Committee (J50305, J50301), China; and the E-Institute of Traditional Chinese Medicine Internal Medicine, Shanghai Municipal Education Commission (E 03008), China.

\section{References}

1. Wang D, Wang J, Huang X, Tu Y and Ni K: Identification of polymethoxylated flavones from green tangerine peel (Pericarpium Citri Reticulatae Viride) by chromatographic and spectroscopic techniques. J Pharm Biomed Anal 44: 63-69, 2007.

2. Hirano T, Abe K, Gotoh M and Oka K: Citrus flavone tangeretin inhibits leukaemic HL-60 cell growth partially through induction of apoptosis with less cytotoxicity on normal lymphocytes. Br J Cancer 72: 1380-1388, 1995.

3. Rooprai HK, Kandanearatchi A, Maidment SL, et al: Evaluation of the effects of swainsonine, captopril, tangeretin and nobiletin on the biological behaviour of brain tumour cells in vitro. Neuropathol Appl Neurobiol 27: 29-39, 2001. 
4. Martinez Conesa C, Vicente Ortega V, Yanez Gascon MJ, et al: Treatment of metastatic melanoma B16F10 by the flavonoids tangeretin, rutin, and diosmin. J Agric Food Chem 53: 6791-6797, 2005.

5. Manthey JA and Guthrie N: Antiproliferative activities of citrus flavonoids against six human cancer cell lines. J Agric Food Chem 50: 5837-5843, 2002.

6. Ishii K, Tanaka S, Kagami K, et al: Effects of naturally occurring polymethyoxyflavonoids on cell growth, $\mathrm{P}$-glycoprotein function, cell cycle, and apoptosis of daunorubicin-resistant T lymphoblastoid leukemia cells. Cancer Invest 28: 220-229, 2010.

7. Morley KL, Ferguson PJ and Koropatnick J: Tangeretin and nobiletin induce G1 cell cycle arrest but not apoptosis in human breast and colon cancer cells. Cancer Lett 251: 168-178, 2007.

8. Pan MH, Chen WJ, Lin-Shiau SY, Ho CT and Lin JK: Tangeretin induces cell-cycle G1 arrest through inhibiting cyclin-dependent kinases 2 and 4 activities as well as elevating Cdk inhibitors p21 and p27 in human colorectal carcinoma cells. Carcinogenesis 23 1677-1684, 2002.

9. Chen KH, Weng MS and Lin JK: Tangeretin suppresses IL-1beta-induced cyclooxygenase (COX)-2 expression through inhibition of p38 MAPK, JNK, and AKT activation in human lung carcinoma cells. Biochem Pharmacol 73: 215-227, 2007.

10. Lust S, Vanhoecke B, Van Gele M, Philippe J, Bracke M and Offner F: The flavonoid tangeretin activates the unfolded protein response and synergizes with imatinib in the erythroleukemia cell line K562. Mol Nutr Food Res 54: 823-832, 2010.

11. Arafa el SA, Zhu Q, Barakat BM, et al: Tangeretin sensitizes cisplatin-resistant human ovarian cancer cells through downregulation of phosphoinositide 3-kinase/Akt signaling pathway. Cancer Res 69: 8910-8917, 2009.

12. Gao J, Senthil M, Ren B, et al: IRF-1 transcriptionally upregulates PUMA, which mediates the mitochondrial apoptotic pathway in IRF-1-induced apoptosis in cancer cells. Cell Death Differ 17: 699-709, 2010.

13. Jiang $\mathrm{XH}$, Wong BC, Lin MC, et al: Functional p53 is required for triptolide-induced apoptosis and AP-1 and nuclear factor- $\kappa \mathrm{B}$ activation in gastric cancer cells. Oncogene 20: 8009-8018, 2001.

14. Linke SP, Clarkin KC and Wahl GM: p53 mediates permanent arrest over multiple cell cycles in response to $\gamma$-irradiation. Cancer Res 57: 1171-1179, 1997.

15. Midgley CA, Owens B, Briscoe CV, Thomas DB, Lane DP and Hall PA: Coupling between gamma irradiation, p53 induction and the apoptotic response depends upon cell type in vivo. J Cell Sci 108: 1843-1848, 1995.

16. Goldstein I, Marcel V, Olivier M, Oren M, Rotter V and Hainaut P: Understanding wild-type and mutant p53 activities in human cancer: new landmarks on the way to targeted therapies. Cancer Gene Ther 18: 2-11, 2011.

17. Dong Y, Ji G, Shi JR, Cao AL, Xie JQ and Wu DZ: Arrest of human gastric cancer AGS cells by tangeretin via cyclinB1 upregulation and inhibition of ERK phosphorylation. Chin Pharmacol Bull 28: 5,2012
18. Cohen GM: Caspases: the executioners of apoptosis. Biochem J 326: 1-16, 1997.

19. Budihardjo I, Oliver H,Lutter M, Luo X and Wang X: Biochemical pathways of caspase activation during apoptosis. Annu Rev Cell Dev Biol 15: 269-290, 1999.

20. Dias N and Bailly C: Drugs targeting mitochondrial functions to control tumor cell growth. Biochem Pharmacol 70: 1-12, 2005.

21. Schulze-Osthoff K, Ferrari D, Los M, Wesselborg S and Peter ME: Apoptosis signaling by death receptors. Eur J Biochem 254: 439-459, 1998.

22. Rao RV, Ellerby HM and Bredesen DE: Coupling endoplasmic reticulum stress to the cell death program. Cell Death Differ 11: 372-380, 2004

23. Zamzami N, Marchetti P, Castedo M, et al: Sequential reduction of mitochondrial transmembrane potential and generation of reactive oxygen species in early programmed cell death. J Exp Med 182: 367-377, 1995.

24. Zamzami N, Marchetti P, Castedo M, et al: Reduction in mitochondrial potential constitutes an early irreversible step of programmed lymphocyte death in vivo. J Exp Med 181: 1661-1672, 1995.

25. Kudla G, Montessuit S, Eskes R, et al: The destabilization of lipid membranes induced by the $\mathrm{C}$-terminal fragment of caspase 8 -cleaved bid is inhibited by the N-terminal fragment. J Biol Chem 275: 22713-22718, 2000

26. Roucou X, Montessuit S, Antonsson B and Martinou JC: Bax oligomerization in mitochondrial membranes requires tBid (caspase-8-cleaved Bid) and a mitochondrial protein. Biochem J 368: 915-921, 2002.

27. Schug ZT, Gonzalvez F, Houtkooper RH, Vaz FM and Gottlieb E: BID is cleaved by caspase- 8 within a native complex on the mitochondrial membrane. Cell Death Differ 18: 538-548, 2011.

28. Vousden KH and Lu X: Live or let die: the cell's response to p53. Nat Rev Cancer 2: 594-604, 2002.

29. Cui Q, Yu JH, Wu JN, et al: P53-mediated cell cycle arrest and apoptosis through a caspase-3-independent, but caspase9-dependent pathway in oridonin-treated MCF-7 human breast cancer cells. Acta Pharmacol Sin 28: 1057-1066, 2007.

30. Lu L, Dong Y, Shi JR and Wu DZ: Tangeretin enhances the proliferation inhibition of 5-fluorouracil in human gastric cancer AGS cells. Chin Pharmacol Bull 27: 760-763, 2011.

31. Bunz F, Dutriaux A, Lengauer C, et al: Requirement for $\mathrm{p} 53$ and $\mathrm{p} 21$ to sustain G2 arrest after DNA damage. Science 282: 1497-1501, 1998.

32. Kurland JF and Meyn RE: Protease inhibitors restore radiationinduced apoptosis to $\mathrm{Bcl}-2$-expressing lymphoma cells. Int $\mathrm{J}$ Cancer 96: 327-333, 2001. 